\title{
EQUIVARIANT IMMERSION AND IMBEDDING UP TO COBORDISM
}

\author{
R. E. STONG ${ }^{1}$
}

\begin{abstract}
This paper determines the smallest possible integer $r$ so that a given manifold with involution is equivariantly bordant to an immersed or imbedded submanifold of some $R^{s} \times R^{r}$ with involution $1 \times(-1)$.
\end{abstract}

1. Introduction. Being given a class $\alpha \in \mathfrak{N}_{n}^{Z_{2}}$ in the cobordism group of manifolds with unrestricted involution, Bix [1] has defined integers $\phi(\alpha)$ and $\psi(\alpha)$ by:

(a) $\phi(\alpha)$ is the smallest integer $r$ for which there is a representative $\left(M^{n}, T\right)$ of $\alpha$ which immerses equivariantly in $R^{s, r}$ for some $s$, where $R^{s, r}=R^{s} \times R^{r}$ with involution $1 \times(-1)$, and

(b) $\psi(\alpha)$ is the smallest integer $r$ for which there is a representative $\left(M^{n}, T\right)$ of $\alpha$ which imbeds equivariantly in $R^{s, r}$ for some $s$.

Being given $\alpha$, the fixed data of $\alpha$ consists of classes $\alpha_{j} \in \mathfrak{N}_{n-j}\left(B O_{j}\right)$. Let $\eta(\alpha)$ be the smallest integer $k$ such that each $\alpha_{j}$ lies in the image of $\mathfrak{N}_{n-j}\left(G_{j, k}\right)$, where $G_{j, k}$ is the Grassmanian of $j$ planes in $R^{k}$.

Note. If $\alpha_{j}=\left[F^{n-j}, \nu^{j}\right] \in \mathfrak{N}_{n-j}\left(B O_{j}\right)$, the condition that $\alpha_{j}$ lie in the image of $\mathfrak{N}_{n-j}\left(G_{j, k}\right)$ is that all Stiefel-Whitney numbers involving $\bar{w}_{i}(v)$ vanish if $i>k-j$.

THEOREM. $\quad \phi(\alpha)=\psi(\alpha)=\eta(\alpha)$.

2. Proof of the Theorem. Clearly $\phi(\alpha) \leqq \psi(\alpha)$ since an imbedding is an immersion, so it suffices to show that $\eta(\alpha) \leqq \phi(\alpha)$ and $\psi(\alpha) \leqq \eta(\alpha)$.

To see that $\eta(\alpha) \leqq \phi(\alpha)$, suppose $\left(M^{n}, T\right)$ represents $\alpha$ and $f:\left(M^{n}, T\right) \rightarrow$ $R^{s, r}$ is an immersion. Then on the $(n-j)$-dimensional component of the fixed set $F^{n-j}$, the normal bundle in $M, v^{j}$, has a complement $\rho$ of dimension $r-j$, for $F^{n-j}$ immerses in $R^{s} \times O$ and $\nu^{j}$ is a subbundle of the pullback of the normal bundle of $R^{s} \times O$ in $R^{s} \times R^{r}$, which is a trivial $r$-plane bundle. Thus $\nu^{j}$ is classified by a map into $G_{j, r}$ and $\eta(\alpha) \leqq r$.

Received by the editors August 8, 1973 and, in revised form, September 11, 1973. AMS (MOS) subject classifications (1970). Primary 57D85, 57D40.

${ }^{1}$ The author is indebted to the National Science Foundation for financial support during this work.

(c) American Mathematical Society 1974 
To see that $\psi(\alpha) \leqq \eta(\alpha)$, let $\alpha$ be given and represent $\alpha_{j}$ by maps $F^{n-j} \rightarrow$ $G_{j, \eta(x)}$. Then the $F^{n-j}$ may be imbedded disjointly in $R^{s}$ for some large $s\left(s>2 n+1\right.$ will suffice) and the normal bundle $\nu^{j}$ has a complement $\rho$ of dimension $\eta(\alpha)-j$ so that $D\left(\nu^{j}\right) \subset D\left(\nu^{j} \oplus \rho\right)$ imbeds (fiberwise) in the trivial bundle $R^{s} \times R^{\eta(\alpha)}$ over $R^{s}$ (in fact in the space $R^{s} \times D^{\eta(\alpha)}$ ). Letting $N$ be the tubular neighborhood of the fixed data given by the union of the $D\left(v^{j}\right), \partial N$ imbeds equivariantly in $R^{s} \times S^{\eta(\alpha)-1}$ or $\partial N / Z_{2}$ imbeds in $R^{s} \times R P(\eta(\alpha)-1)$. The map $g: \partial N / Z_{2} \rightarrow R^{s} \times R P(\eta(\alpha)-1)$ bounds in $R^{s} \times R P(\infty)$, that being the condition that the collection of $\alpha_{j}$ come from some $\alpha$, and $\mathfrak{N}_{*}(R P(i)) \rightarrow \mathfrak{N}_{*}(R P(\infty))$ is monic, so $g$ bounds. For $s$ sufficiently large $(s>2 n+1$ being sufficient) $g$ bounds an imbedded manifold with boundary $h: W \rightarrow R^{s} \times R P(\eta(\alpha)-1)$, and taking double covers $\tilde{h}: \tilde{W} \rightarrow R^{s} \times S^{\eta(\alpha)_{-1}}$ is an imbedding. Joining $N$ and $\tilde{W}$ along their common boundary gives a closed manifold $M^{n}$, with the involution $T$ induced by -1 in the fibers of $D\left(v^{j}\right)$ and the involution of the double cover on $\tilde{W}$, and with an imbedding $f:(M, T) \rightarrow R^{s, \eta(\alpha)}$ induced by $\tilde{h}$ and the imbedding of $D\left(\nu^{j}\right)$ in $R^{s} \times D^{\eta(\alpha)}$. Since the fixed data of $\left(M^{n}, T\right)$ is given by the $F^{n-j}$ and $\nu^{j},\left(M^{n}, T\right)$ represents $\alpha$, and so $\psi(\alpha) \leqq \eta(\alpha)$.

Note. Using a tubular neighborhood of $\partial N / \bar{Z}_{2}$ in $W$, one may make $f$ smooth.

3. Remarks. One should compare this with Bix's result which computes $\eta(\alpha)$ for $\alpha$ in a certain base for $\mathfrak{N}_{*}^{Z_{2}}$ over $Z_{2}$.

To describe the base, one lets $R P(n)$ have the involution

$$
T\left(\left[x_{0}, \cdots, x_{n}\right]\right)=\left[-x_{0}, x_{1}, \cdots, x_{n}\right],
$$

and being given $M$ with involution $T, \Gamma(M)$ is $S^{1} \times M$ with $(z, m)$ identified with $(-z, T m)$ and with involution induced by $(z, m) \rightarrow(\bar{z}, m)$, where $\bar{z}$ is the conjugate of the complex number $z$. The base then consists of all classes $\alpha=\Gamma^{i} R P\left(n_{1}\right) \times R P\left(n_{2}\right) \times \cdots \times R P\left(n_{k}\right) \times M^{m}$, with the diagonal action, $M$ having trivial action and $[M]$ forming a base for $\mathfrak{N}_{*}$, and each $n_{j}>1$

The fixed set of $T$ on $R P(n)$ is $R P(0)$ with trivial normal bundle, classified in $G_{n, n}$ and $R P(n-1)$ with the universal line bundle classified in $G_{1, n}$. The fixed data of $\Gamma(M)$ is obtained by adding a trivial line bundle to the fixed set of $M$, i.e. if $F^{n-j} \rightarrow G_{j, k}$, one is transformed to $G_{j+1, k+1}$ and by taking $M$ with a trivial line bundle in $G_{1,1}$. For a product, the fixed data is classified via the Whitney sum maps $G_{j, k} \times G_{m, n} \rightarrow G_{j+m, k+n}$.

It is then immediate that for $\alpha=\Gamma R^{i} P\left(n_{1}\right) \times \cdots \times R P\left(n_{k}\right) \times M^{m}$, $\eta(\alpha) \leqq i+n_{1}+\cdots+n_{k}$.

The fixed component of least dimension in $\Gamma^{i} R P\left(n_{1}\right) \times \cdots \times R P\left(n_{k}\right) \times$ $M^{m}$ is $M^{m}$ with trivial normal bundle of dimension $j=i+n_{1}+\cdots+n_{k}$, 
and if $[M] \neq 0$ in $\mathfrak{N}_{m}$, this gives a nontrivial class in $\mathfrak{N}_{m}\left(G_{j, j}\right) \subset \mathfrak{N}_{m}\left(B O_{j}\right)$, so $\eta(\alpha) \geqq i+n_{1}+\cdots+n_{k}$. Thus, one has Bix's theorem:

If $\alpha=\Gamma^{i} R P\left(n_{1}\right) \times R P\left(n_{2}\right) \times \cdots \times R P\left(n_{k}\right) \times M^{m}$ with $[M] \neq 0$ in $\mathfrak{N}_{m}$, then $\eta(\alpha)=i+n_{1}+\cdots+n_{k}$.

\section{REFERENCE}

1. Michael C. Bix, $Z_{2}$-equivariant immersions and embeddings up to cobordism, Proc. Amer. Math. Soc. 44 (1973), 203-207.

Department of Mathematics, University of Virginia, Charlottesville, Virginia 22903 